\title{
HIGH-FREQUENCY LARGE-DEFLECTION ELECTROSTATIC DIAPHRAGM ACTUATORS WITH MAXIMIZED VOLUME DISPLACEMENT
}

Seow Yuen Yee, Rebecca L. Peterson, Luis P. Bernal and Khalil Najafi ${ }^{*}$

Center for Wireless Integrated MicroSensing and Systems $\left(W I M S^{2}\right)$, University of Michigan, Ann Arbor, Michigan USA

\section{ABSTRACT}

This paper demonstrates electrostatic (ES) actuators with high frequency diaphragms $(>90 \mathrm{kHz})$, large deflection $(>8 \mu \mathrm{m})$, high volume displacement, and reliable operation using a new fabrication process and device structure. Electrode gaps of up to $9.58 \mu \mathrm{m}$ have been fabricated with a filleted electrode shape which allows the pulled-in diaphragm to follow the curved profile of the electrode, displacing $100 \%$ of the air gap volume. The filleted electrode actuators have been dynamically actuated for over 212 hours without any diaphragms breaking or sticking. In contrast flat electrode diaphragms broke after $<2$ minutes of actuation.

\section{INTRODUCTION}

Many MEMS applications require high-deflection $(>5 \mu \mathrm{m})$, high-frequency $(>50 \mathrm{kHz})$, and low-power diaphragm actuators which displace a large air volume. Examples include micropropulsion systems (high-velocity air jets), micro-pumps (high flow rate), micro-speakers (high sound pressure), and microcoolers (large flow volume). Diaphragm actuation methods include electrostatic (ES) [1-3], electromagnetic [4], piezoelectric [5] and thermo-pneumatic [6]. Typically electromagnetic and piezoelectric actuators face challenges in device miniaturization, while electromagnetic and thermo-pneumatic actuators are limited by slow response time or low actuation frequency. Therefore we pursue ES actuators for high-frequency, high-deflection microactuation.

High frequency operation of parallel plate capacitive ES diaphragm actuators requires small-mass and high-stiffness diaphragms with large intrinsic stress. Large-deflection ES diaphragm actuators induce large extrinsic stress and require large drive voltages, inducing high electric fields. In addition, the microfabrication of large air gaps is not easy. The combination of large mechanical stress (causing fracture), high electric field (degrading reliability and lifetime), and fabrication difficulties has prevented these actuators from being widely utilized. Curved or zipper ES actuators have been made [1-3], but they do not provide the high frequency, large deflection, reliability, and maximum volume displacement needed.

Previous ES actuators fabricated by our group achieved deflection of $6 \mu \mathrm{m}$ [2] and $8.8 \mu \mathrm{m}$ [3], but only a portion of the total air gap volume was displaced. Here we present an ES diaphragm actuator with large deflection $(>8 \mu \mathrm{m})$, maximized volume displacement and reliable actuation, made using a much simpler and more robust fabrication process. We compare the performance of two different electrode designs, flat and filleted.

\section{FABRICATION}

\section{Flat Electrode}

Arrays of ES actuators (Figure 1 (Left)) are fabricated with a simple three-mask process (Figure 2a). First, an oxide-nitrideoxide stack is deposited by LPCVD on a blank silicon wafer. The deposited dielectric stack has a total thickness of $\sim 1.75 \mu \mathrm{m}$ and intrinsic stress of 32 or $74 \mathrm{MPa}$ for membrane resonance frequencies of $64 \mathrm{kHz}$ and $97 \mathrm{kHz}$, respectively. Next, a $\sim 10 \mu \mathrm{m}$ layer of sacrificial photoresist (PR) AZ9260 is spun and patterned
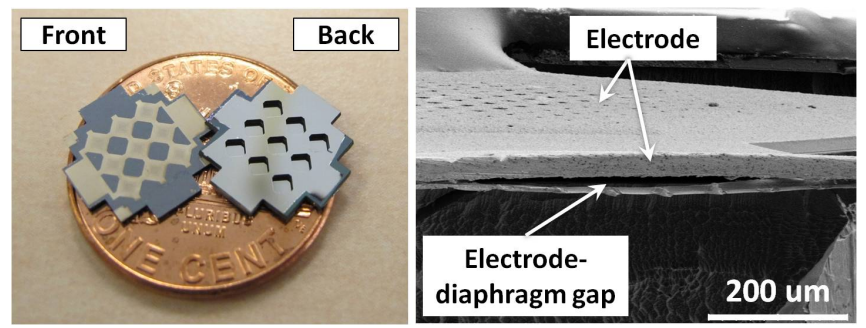

Figure 1: (Left) Fabricated device with an array of nine ES diaphragm actuators, (Right) SEM of flat electrode cross section.

to define the air gap between the diaphragm and electrode. A seed layer of chrome/gold is sputtered over the whole wafer, followed by spinning and patterning of a $>20 \mu \mathrm{m}$ photoresist mold to shape the electroplated nickel electrode. After electroplating, the photoresist mold, seed layer, and sacrificial photoresist are sequentially etched and removed. Finally, the diaphragm is released by deep reactive ion etching (DRIE) from the backside and a thin layer of aluminum is sputtered on backside to form the second electrode of the parallel plate capacitor. Figure 1(Right) shows an SEM of the cross section of a flat electrode actuator.
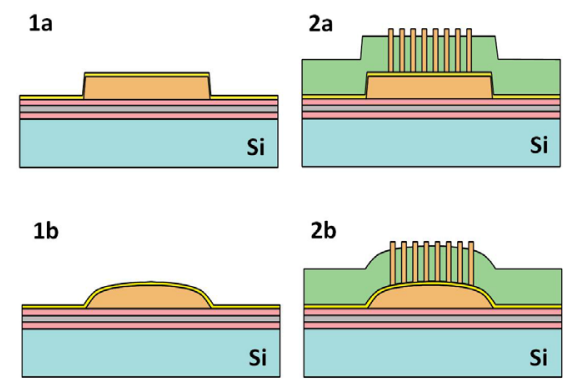

LPCVD ox/ni/ox Pattern sacrificial PR Reflow PR (only 1b)

Pattern PR mold

Sputter $\mathrm{Cr} / \mathrm{Au}$
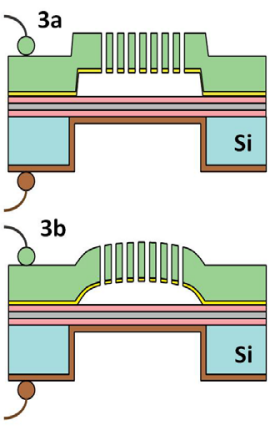

Remove PR mold Etch $\mathrm{Cr} / \mathrm{Au}$ Remove sacrificial PR Sputter Cr/Al from back DRIE backside etch

Figure 2: Fabrication process for (a) flat electrode and (b) filleted electrode actuators.

\section{Filleted Electrode}

Filleted electrodes were designed to maximize volume displacement and improve the reliability of the large-gap ES actuator by reducing the large electric field and mechanical stress at the edges of the diaphragm. The filleted electrodes are fabricated with a similar fabrication process but with an extra step: re-shaping of the sacrificial photoresist through a reflow process (Figure 2b). The additional PR reflow step is modified from [7], in which the wafer is turned upside down and exposed to acetone vapor for 8-10 minutes to reshape the PR through gravity and surface tension. The reflow process duration is optimized to shape the PR into a dome while maintaining its base pattern (square, 
hexagon, etc.) as shown in Figure 3. The reflow process increases the PR thickness at the center (Table 1), so the initial PR layer is made thinner to achieve the same final gap as in the flat electrode (Figure 4). Figure 5 shows microscope images of the flat and filleted electrode before and after electroplating. We note that a thicker PR layer could be used in the future for greater volume displacement.
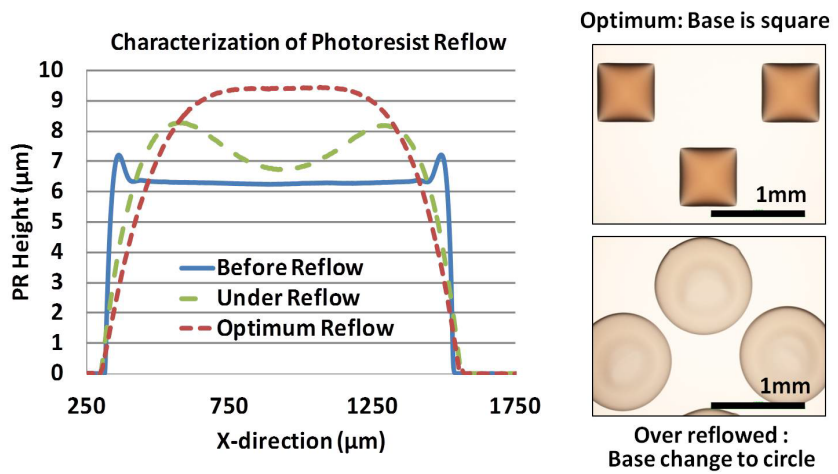

Figure 3: (Left) Filleted sacrificial PR profile under insufficient and optimal reflow; and (right) micrographs showing excessive reflow of base shape from square to circle-like.

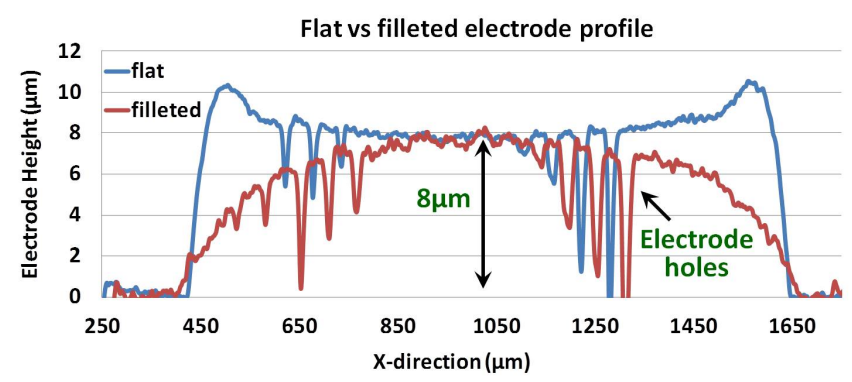

Figure 4: Comparison of flat and filleted electrode profiles with the same center gap spacing.

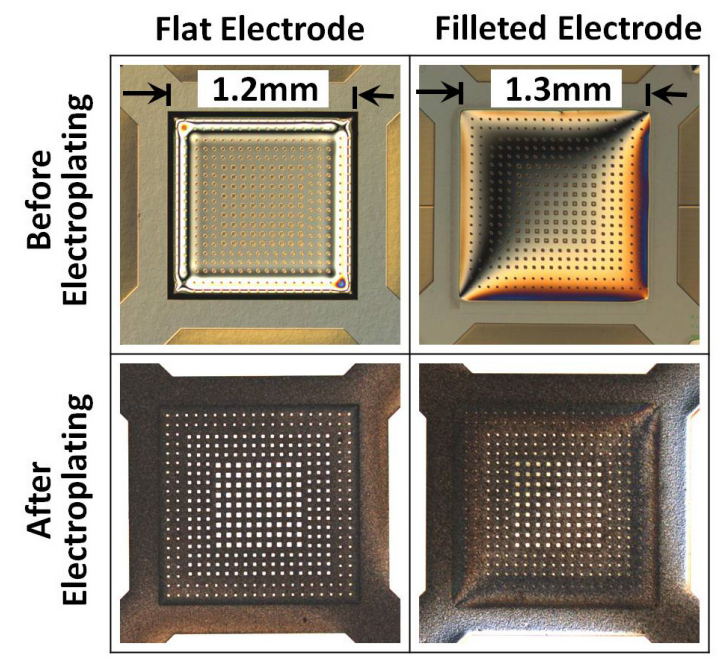

Figure 5: Microscope images before and after electroplating (front view) for flat and filleted electrode.
Table 1: Performance of flat vs. filleted electrode actuators.

\begin{tabular}{|c|c|c|c|}
\hline Device Parameters & \multicolumn{2}{|c|}{ Flat Electrode } & Filleted Electrode \\
\hline Diaphram resonance frequency & $64 \mathrm{kHz}$ & $97 \mathrm{kHz}$ & $97 \mathrm{kHz}$ \\
\hline Diaphragm residual stress & $32 \mathrm{MPa}$ & $74 \mathrm{MPa}$ & $74 \mathrm{MPa}$ \\
\hline Diaphragm area & \multicolumn{2}{|c|}{$1.2 \mathrm{~mm} \times 1.2 \mathrm{~mm}$} & $1.3 \mathrm{~mm} \times 1.3 \mathrm{~mm}$ \\
\hline Diaphragm thickness & $1.46 \mu \mathrm{m}$ & $1.74 \mu \mathrm{m}$ & $1.74 \mu \mathrm{m}$ \\
\hline Sacrificial PR height before reflow & \multirow{2}{*}{$7.9 \mu \mathrm{m}$} & \multirow{2}{*}{$8.33 \mu \mathrm{m}$} & $6.2 \mu \mathrm{m}$ \\
\hline Average gap at center & & & $8.14 \mu \mathrm{m}$ \\
\hline $\begin{array}{l}\text { Applied signal for diaphragm pull- } \\
\text { in }\end{array}$ & $\begin{array}{l}(10 \mathrm{kHz}) \\
\pm 210 \mathrm{~V}\end{array}$ & $\begin{array}{l}\text { (DC) } 337 \mathrm{~V} \\
\text { (AC) N/A }\end{array}$ & $\begin{array}{l}(1 \mathrm{kHz}) \pm 240 \mathrm{~V} \\
(10 \mathrm{kHz}) \pm 279 \mathrm{~V}\end{array}$ \\
\hline Air gap volume (calculated) & \multicolumn{2}{|c|}{$11.52 \mathrm{e}^{-12} \mathrm{~m}^{3}$} & $9.7 \mathrm{e}^{-12} \mathrm{~m}^{3}$ \\
\hline Volume displaced (calculated) & \multicolumn{2}{|c|}{$8.65 \mathrm{e}^{-12} \mathrm{~m}^{3}$} & $9.7 \mathrm{e}^{-12} \mathrm{~m}^{3}$ \\
\hline Reliable actuation & $<2 \min$ & - & $1 \mathrm{hr}$ \\
\hline
\end{tabular}

\section{TEST RESULTS}

\section{Flat Electrode}

The flat electrode $97 \mathrm{kHz}$-diaphragms with a $\sim 8.3 \mu \mathrm{m}$ gap pull-in at $\sim 337 \mathrm{~V} \mathrm{DC}$, and easily break due to the large electric field and mechanical stress at the edges. For the $64 \mathrm{kHz}-$ diaphragms with flat electrodes (7.4-8.22 $\mu \mathrm{m}$ center gap), pull-in happens for pulse waveforms of $\pm 210 \mathrm{~V}$ at $10 \mathrm{kHz}$. The left columns of Figures 6 and 7 show that the collapsed diaphragms displace $75 \%$ of the total gap volume. Only six of the nine diaphragms are pulled down and one or more diaphragms start to break after 2 seconds of actuation, and all of the diaphragms break after only $\sim 2$ minutes of actuation (left column of Figure 8 ).

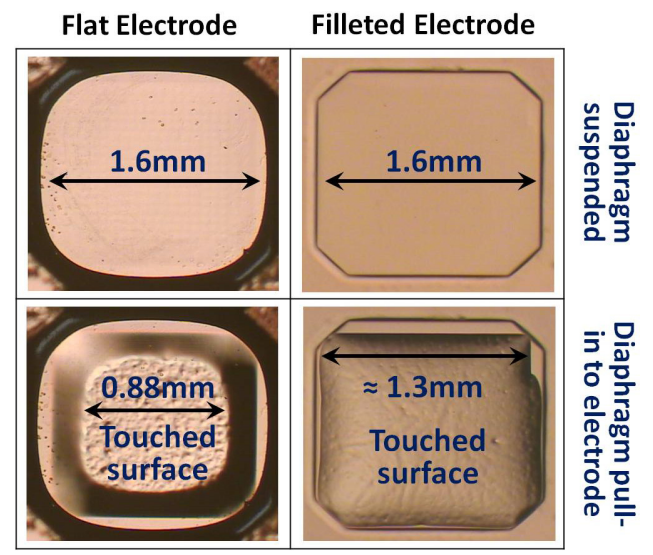

Figure 6: Back view of diaphragm pull-in shape for flat lleft column) and filleted electrodes (right column).

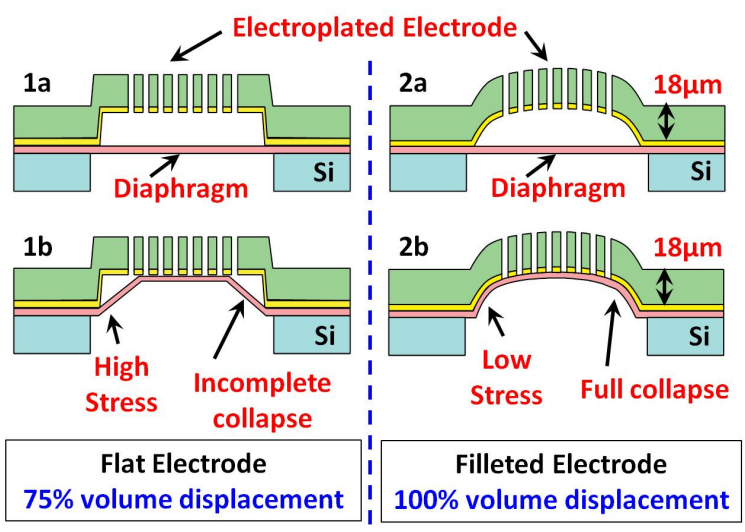

Figure 7: Cross sectional diagram: flat vs. filleted electrode. 


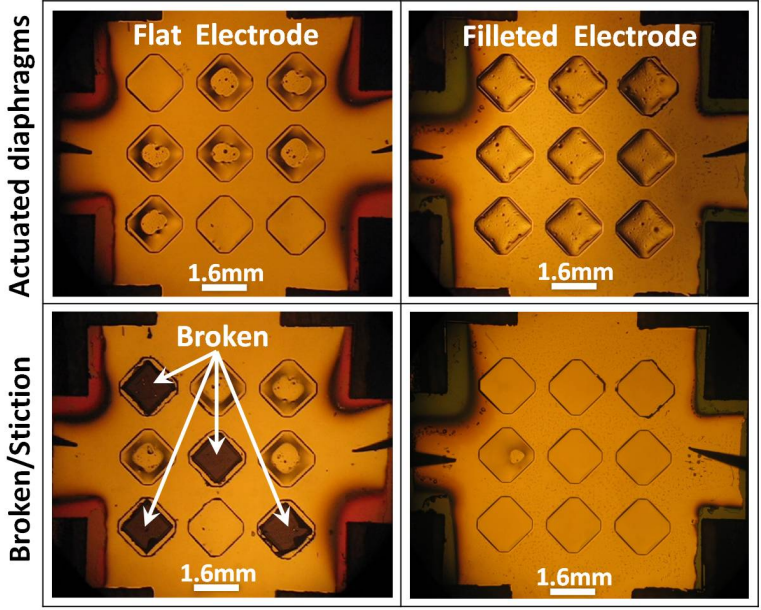

Figure 8: Long Term Test 1: Back view. (Left top) Only six of nine flat electrode diaphragms pull in. (Right to) All filleted electrode diaphragms pull-in and are held against nickel electrode. (Left bottom) One or more diaphragms break after $\sim 2$ minutes actuation. (Right bottom) No diaphragms break after 1 hour actuation.

\section{Filleted Electrode}

With a slightly modified electrode, the $97 \mathrm{kHz}$-diaphragms with filleted electrode $(7.7-9.58 \mu \mathrm{m}$ center gap) pull in at pulse waveform of $\pm 240 \mathrm{~V}$ at $10 \mathrm{kHz}$ (Table 1). The filleted-electrode diaphragm hugs the curved profile of the electrode, displacing $\sim 100 \%$ of the air gap volume (right columns of Figure 6 and 7).

In order to investigate diaphragm deflection, a Polytec laser vibrometer is used to measure dynamic deflection. The laser is focused on the center diaphragm of the actuator array. The diaphragm is actuated from $1 \mathrm{kHz}$ to $14 \mathrm{kHz}$ at varying voltages and duty cycles. (The maximum frequency is limited by the current drive circuitry.) We find that a duty cycle of $20 \%$ provides the best actuation for maximum deflection and triangle-like movement. At voltages greater than the pull-in voltage and with long duty cycles, the diaphragm collapses onto the electrode and sticks to the electrode. Figure 9 shows a drive signal at $10 \mathrm{kHz}$ with $20 \%$ duty cycle and the corresponding diaphragm displacement detected with the laser vibrometer.
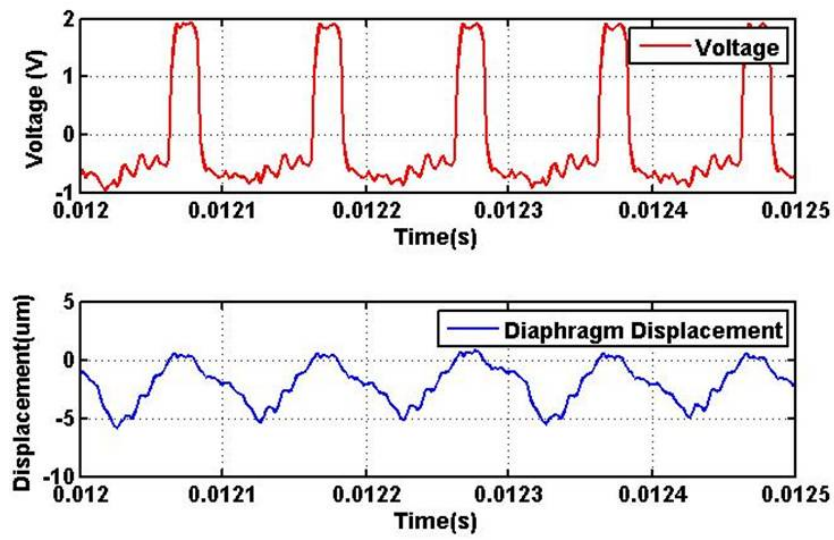

Figure 9: (top) Voltage applied across the actuator array (scaled by a factor of 1/97). (Bottom) Measured displacement of the center diaphragm, as detected by the vibrometer.

\section{Reliability Test of Filleted Electrodes}

Reliability tests with different degrees of mechanical and electrical stress were performed to investigate the device limits. For the worst-case scenario, the diaphragm is collapsed and held to the bottom electrode for a long time. This test injects charge into the dielectric layer from both the bottom and top electrodes, similar to the tests in [8]. In addition, the diaphragm is maximally deflected thus inducing maximum mechanical stress on the diaphragm edges. The diaphragm is held to the electrode with a pulse waveform of $10 \mathrm{kHz}, \pm 279 \mathrm{~V}$ and $50 \%$ duty cycle. The $50 \%$ duty cycle and large actuation voltage cause the diaphragm to be held to the electrode continually. After some time, the voltage is completely removed to investigate whether the diaphragm returns to its release position.

In this test, all nine diaphragms of the actuator array are held against the electrode for $>30$ minutes with no stiction. Only one filleted electrode diaphragm exhibits stiction after 1 hour (right bottom image of Figure 8). This is at least 30 times better than the flat electrode design, where the diaphragm broke beyond repair after $\sim 2$ minutes. Of all three reliability tests conducted, this test shows the shortest reliable actuation time. This test was used to model the worst-case scenario in which charge is injected from both the top and bottom electrodes [9].

The second reliability test involves repeatedly pulling in the diaphragm towards the nickel electrode and then releasing it. This test is designed to investigate its fatigue and DC drift during dynamic operation. In this test, the duration of time when the diaphragm is in contact with the electrode is minimized compared to the first test, so that charging from the nickel electrode is minimized. A $20 \%$ duty cycle is used to minimize the contact time, creating triangle-like dynamic movement for all diaphragms, as shown in Figure 9. For the long-term test, a calibrated microphone is used to measure the acoustic pressure. Tests with various pulse waveforms are performed and sound pressure from the combined movement of the array of diaphragms is measured by a calibrated microphone positioned $\sim 1.5 \mathrm{~cm}$ away from the array. The long-term test is done using the conditions under which maximum sound pressure is detected, with a pulse waveform of $10 \mathrm{kHz}$, duty cycle of $20 \%$ and voltage across the device of $+242.5 \mathrm{~V}$ to $-48.5 \mathrm{~V}$, for minimal charging effects.

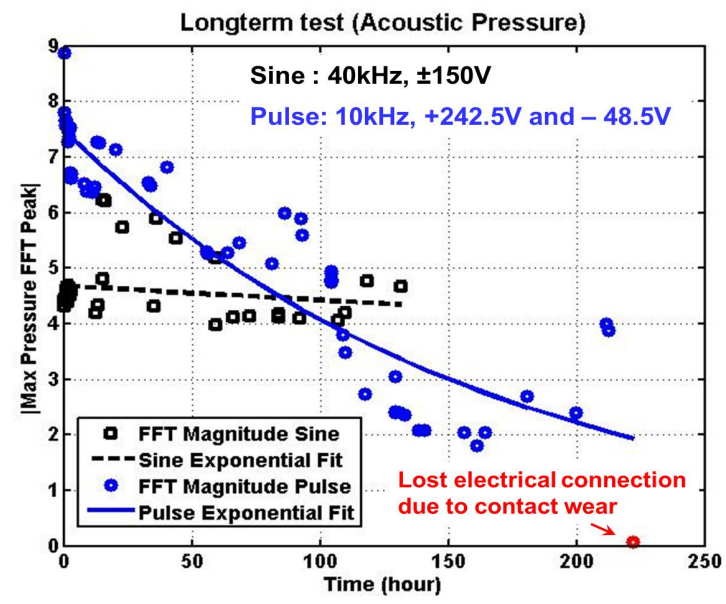

Figure 10: Long term test 2 (blue circle): 20\% duty cycle pulse wave actuation. Sound pressure falls to zero after $\sim 212$ hour due to loss of electrical connection due to damage of the aluminum metal layer (top electrode). Long term test 3 (black square): Sinusoidal actuation, showing a much slower charging effect compared to test 2 . 
The FFT of the acoustic pressure is obtained and the magnitude of the maximum peak of the FFT is plotted with respect to time (Figure 10 (blue circles)). The maximum FFT peak is detected at $110 \mathrm{kHz}$, which is near the $97-\mathrm{kHz}$ resonance frequency of the diaphragm even though it is driven with a $10 \mathrm{kHz}$ pulse waveform. The fluctuations in the detected acoustic pressure might be related to the variable humidity and temperature of the environment during the day or night. For example, higher acoustic pressure corresponding to higher diaphragm deflection is observed at night while lower acoustic pressure is observed during day time. We observe charging effects as the acoustic pressure decays exponentially with time. The charge accumulation in the dielectric of the diaphragm is hypothesized to create DC deflection or DC drift and thus reduces dynamic motion. The time constant for the charging effect is 164 hours. The lower duty cycle used in this test compared with the previous test (in which stiction was observed after $<1$ hour), results in much longer device operation. The lower duty cycle reduces the contact time of the diaphragm to the bottom electrode, which should minimize charging effects as described in [9]. After more than 212 hours, the device stops working when the electrical connection to the top electrode is broken due to damage of the aluminum metal layer under the probe tip. The device can be actuated again if the probe is moved to a different spot, with similar performance, as shown by the first data point in Figure 11. In fact if higher voltage is then applied, the acoustic pressure increases above the initial values before the long-term test began. However, after $\sim 7$ minutes of actuation at this larger voltage, one diaphragm collapses and sticks to the nickel electrode, causing a decrease in acoustic pressure (last point in Figure 11).

In the third reliability test, the diaphragm is driven with a much higher frequency in a sinusoidal fashion $(\sim 80 \mathrm{kHz}$ deflection). A sine waveform at $40 \mathrm{kHz}$ and $\pm 150 \mathrm{~V}$ is applied, plotted in Figure 10 (black squares). The FFT peak frequency is detected at $80 \mathrm{kHz}$. The applied voltage is $\sim \pm 150 \mathrm{~V}$ which is limited by the power amplifier. The array was actuated reliably for greater than 131 hours without significant deterioration in performance. The charging time constant for the sine test is 1754 hours, which is approximately 10.7 times that found using the pulse waveform in the second test. This may be because the second test is performed at higher voltage, close to unipolar waveform and with faster rise and fall time than the sine test, leading to more charging effects during actuation. During the test, there are a couple higher acoustic pressure data points which deviate from the exponential trend. We attribute these jumps in acoustic pressure to partial actuation of certain diaphragm(s) in the actuator array.

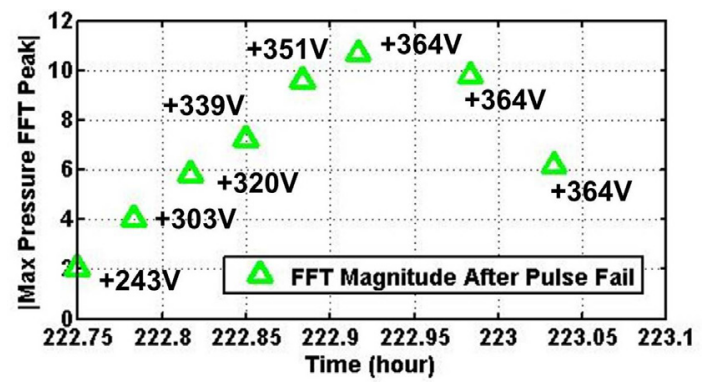

Figure 11: Continuation of long term test 2 after electrical contact is made at a different position. For reference, as plotted in Figure 10, the last measured pressure peak before the probe pad failure was 3.9, at an input voltage of $+243 \mathrm{~V}$.

No diaphragms broke during any of the three reliability tests. The stiction of the diaphragm from the first reliability test releases on its own after a few days. In comparison, the flat electrode diaphragm broke after $<2$ minutes actuation.

\section{CONCLUSIONS}

We have demonstrated a new fabrication process to form flat and filleted large-gap $(>8 \mu \mathrm{m})$ electrostatic actuators. Tests show that the filleted electrodes displace almost $100 \%$ of the air gap volume and are much more reliable than the flat electrodes due to reduction of the electric field and mechanical stress at the edges of the diaphragm. The reliability of the filleted electrode diaphragms has been investigated through several long-term tests. Using a $20 \%$ duty cycle pulse wave to repeatedly pull in the diaphragm, the actuators worked continuously for more than 200 hours until probe pad failure stopped the test. We expect that future tests will show much longer time-to-failure. These high-frequency, largedeflection ES actuators with maximized volume displacement can be used in a variety of microsystems.

\section{ACKNOWLEDGEMENT}

This project is supported by DARPA-CSVMP/HiVac program under grant \#W31P4Q-09-1-0011 and was performed in part at Lurie Nanofabrication Facility (LNF). Travel support has been generously provided by the Transducer Research Foundation.

\section{REFERENCES}

[1] W. van der Wijngaart, H. Ask, P. Enoksson, G. Stemme, “A high-stroke, high pressure electrostatic actuator for valve applications," Sensors and Actuators A: Physical, 100, 264271 (2002).

[2] T.K. Chou, K. Najafi, M.O. Muller, L.P. Bernal, P.D. Washabaugh, "High-density micromachined ejector array for micro propulsion," IEEE Transducers Conference, 2001-2, 890 (2001).

[3] H. Kim, A.H. Jauregui, C. Morrison, K. Najafi, L.P. Bernal, P.D. Washabaugh, "Low-power electrostatic microthruster for propulsion based on Helmholtz-resonance," IEEE MEMS Conference, 2007, 127 (2007).

[4] K. H. Kim, H. J. Yoon, O. C. Jeong, S. S. Yang, "Fabrication and test of a micro magnetic actuator", Sensors and Actuators A 117 (2005) 8-16.

[5] E. E. Aktakka, H. Kim, K. Najafi, "Wafer level fabrication of high performance MEMS using bonded and thinned bulk piezoelectric substrates", Proceedings of Transducers 2009, 849-852.

[6] O. C. Jeong, S. W. Park, S. S. Yang, J. J. Pak, "Fabrication of a peristaltic PDMS micropump", Sensors and Actuators A123-124 (2005) 453-458.

[7] T. Abe, H. Shimamoto, X. Li, "Miniaturization of spherically contoured rectangular AT-cut quartz-crystal resonators by using reactive ion etching," Japanese Journal of Applied Physics, 45, 5283 (2006).

[8] N. Tavassolian, M. Koutsoureli, E. Papandreou, G. Papaioannou, "The effect of silicon nitride stoichiometry on charging mechanisms in RF-MEMS capacitive switches", IEEE Transactions on Microwave Theory and Techniques, 57, 12 (2009).

[9] Z. Peng, X. Yuan, J. C. M. Hwang, D. Forehand, C. L. Goldsmith, "Top vs bottom charging of the dielectric in RF MEMS capacitive switches", Proceedings of Asia-Pacific Microwave Conference 2006, 1535-1538.

\section{CONTACT}

*K. Najafi, tel: +1-734-763-6650; najafi@umich.edu 